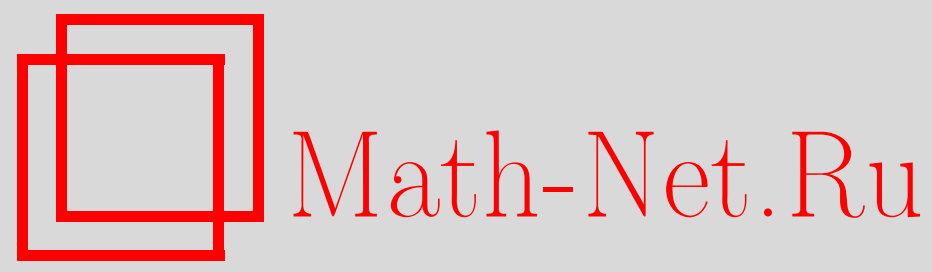

Л. Я. Савельев, С. В. Балакин, Комбинаторное вычисление моментов характеристик серий в троичных марковских последовательностях, Дискрет. матем., 2011, том 23, выпуск 2, 76-92

DOI: https://doi.org/10.4213/dm1143

Использование Общероссийского математического портала Math-Net.Ru подразумевает, что вы прочитали и согласны с пользовательским соглашением http://www . mathnet.ru/rus/agreement

Параметры загрузки:

IP: 52.23 .180 .231

26 апреля 2023 г., 03:52:45 


\title{
Комбинаторное вычисление моментов характеристик серий в троичных марковских последовательностях
}

\author{
() 2011 г. Л. Я. Савельев, С. В. Балакин
}

\begin{abstract}
Описываются маргинальные и совместные распределения чисел значений и чисел серий в троичных марковских последовательностях. Сравнительная простота таких последовательностей позволяет с помощью прямых комбинаторных вычислений вывести точные формулы для средних значений, дисперсий и ковариаций.

Работа выполнена при поддержке Российского фонда фундаментальных исследований, проект 10-01-00384.
\end{abstract}

\section{1. Введение}

Предлагаемая статья продолжает работу авторов [1] о совместных распределениях чисел значений и чисел серий в двоичных марковских последовательностях. В качестве важного частного случая двоичные и троичные марковские последовательности выделяются во многих работах, в которых рассматриваются марковские последовательности с конечным множествам значений [2]-[10]. Двоичные и троичные марковские последовательности наиболее просты, и для распределений различных их характеристик удается получать сравнительно простые точные формулы, поддающиеся анализу и пригодные для вычислений. Эти последовательности широко используются в различных прикладных исследованиях. Совместные распределения различных характеристик серий в двоичных и троичных марковских последовательностях представляют особый интерес. Они позволяют конкретизировать и лучше понять многие общие свойства структуры дискретных марковских последовательностей и имеют важные приложения. В статье описываются совместные распределения различных комбинаций чисел значений и их серий. Выводятся точные формулы для средних значений, дисперсий и ковариаций.

\section{2. Постановка задачи}

2.1. Числа значений и серий из них на конечном отрезке дискретной марковской последовательности не зависят от природы ее значений, и их можно заменить номерами. Рассмотрим марковскую последовательность $\xi$ случайных переменных $\xi(k), k \geqslant 0, \mathrm{c}$ множеством значений $C=\{1, \ldots, \omega\}=\{1,2,3\}$, начальным вектором $A$ и переходной 
матрицей $Q$ :

$$
A=\left(a_{\alpha}\right)=\left(a_{1}, a_{2}, a_{3}\right), \quad Q=\left(q_{\alpha \beta}\right)=\left(\begin{array}{lll}
q_{11} & q_{12} & q_{13} \\
q_{21} & q_{22} & q_{23} \\
q_{31} & q_{32} & q_{33}
\end{array}\right)
$$

где

$$
\begin{aligned}
& a_{\alpha} \geqslant 0, \quad \sum_{\alpha} a_{\alpha}=1, \\
& q_{\alpha \beta} \geqslant 0, \quad \sum_{\beta} q_{\alpha \beta}=1, \quad \alpha, \beta \in C .
\end{aligned}
$$

В рассматриваемом случае $\omega=3$ иногда удобно выделять вероятности $p=q_{11}, q=q_{22}$, $r=q_{33}$, сохранения состояния $1,2,3$ при переходе, вводить относительные коэффициенты $\alpha=q_{12} /\left(1-q_{11}\right), \beta=q_{23} /\left(1-q_{22}\right), \gamma=q_{31} /\left(1-q_{33}\right)$ циклических изменений состояний при переходах $1 \rightarrow 2,2 \rightarrow 3,3 \rightarrow 1$ и записывать переходную матрицу в виде

$$
Q=\left(\begin{array}{ccc}
p & (1-p) \alpha & (1-p)(1-\alpha) \\
(1-q)(1-\beta) & q & (1-q) \beta \\
(1-r) \gamma & (1-r)(1-\gamma) & r
\end{array}\right)
$$

Подчеркнем, что здесь $\alpha, \beta, \gamma$ уже не индексы, а коэффициенты. Для этой записи нужно, чтобы $q_{11}, q_{22}, q_{33}<1$. Если $a_{3}=\beta=0, \alpha=r=1$, то последовательность эквивалентна двоичной, рассматривавшейся в [1]. Естественно выделяются также случаи $\alpha=\beta=\gamma=1$ и $p=q=r=0$. В первом случае значение сохраняется или изменяется циклически, а во втором изменяется с вероятностью 1. Выделяются также случай симметричной матрицы $Q$. Условие $q_{\alpha \beta}=q_{\beta \alpha}$ делает матрицу $Q$ бистохастической.

Обозначим $\tau=\operatorname{tr} Q$ след матрицы $Q, v=(\tau-1) / 2$ ее нормированный след, $\Delta=\operatorname{det} Q$ ее определитель, $\Delta_{\alpha \beta}$ алгебраическое дополнение элемента $q_{\alpha \beta}, I$ единичную $3 \times 3$ матрицу. Они составляют матрицу

$$
M=\left(\begin{array}{lll}
\Delta_{11} & \Delta_{12} & \Delta_{13} \\
\Delta_{21} & \Delta_{22} & \Delta_{23} \\
\Delta_{31} & \Delta_{32} & \Delta_{33}
\end{array}\right)
$$

Как легко проверить,

$$
\operatorname{det}(\lambda I-Q)=(\lambda-1)\left(\lambda^{2}-2 \nu \lambda+\Delta\right)
$$

Характеристическими для матрицы $Q$ служат числа

$$
\lambda_{1}=1, \quad \lambda_{2}=v+\mu, \quad \lambda_{3}=v-\mu,
$$

где $\mu=\sqrt{v^{2}-\Delta} \geqslant 0$.

Заметим, что $\lambda_{2}+\lambda_{3}=2 v, \lambda_{2} \cdot \lambda_{3}=\Delta$. Матрица $Q$ стохастическая, поэтому $-1 / 2 \leqslant v \leqslant 1,\left|\lambda_{2}\right| \leqslant 1,\left|\lambda_{3}\right| \leqslant 1$ и $\left|\lambda_{2}\right|<1,\left|\lambda_{3}\right|<1$ при $q_{\alpha \beta}>0$ (см. стр. 4 в [4]). Следовательно, $|\Delta| \leqslant 1$ и $|\Delta|<1$ при $q_{\alpha \beta}>0$.

Выделим особые случаи. Нетрудно проверить, что

$$
\begin{gathered}
\lambda_{2}=\lambda_{3} \Longleftrightarrow \mu=0 \Longleftrightarrow \Delta=v^{2} \Longleftrightarrow|v|=\sqrt{\Delta} \Longleftrightarrow \lambda_{2}=\lambda_{3}=\sqrt{\Delta}, \\
\lambda_{2}=1 \Longleftrightarrow \mu=1-v \Longleftrightarrow \Delta=2 v-1 \Longleftrightarrow v=(\Delta+1) / 2, \\
\lambda_{3}=1 \Longleftrightarrow v=v-1 \Longleftrightarrow v=1 \Longleftrightarrow \tau=3 \Longleftrightarrow Q=I .
\end{gathered}
$$


Равенства $1=\lambda_{2}=\lambda_{3}$ верны, если и только если $Q=I$. Условия $v \neq 1, \Delta \neq 2 v-1$, $\Delta \neq v^{2}$ исключают существование равных характеристических чисел. Легко также проверить, что эти условия исключают также равенства $\left|\lambda_{2}\right|=1,\left|\lambda_{3}\right|=1$. Из теоремы Гершгорина [11] следует, что для этих равенств необходимо условие $v=-1 / 2$. Из неравенств $\left|\lambda_{2}\right|<1,\left|\lambda_{3}\right|<1$ следует неравенство $|\Delta|<1$.

Замечание 1. Сформулированные условия обеспечивают регулярность рассматриваемой марковской последовательности. Как правило, в дальнейшем без оговорок рассматривается регулярная марковская последовательность. Исключения будут очевидны или будут специально оговариваться. В выкладках с определителем $\Delta$ часто неявно предполагается, что $\Delta \neq 0$.

2.2. Для вычисления степеней $Q^{n}$ переходной матрицы $Q$ проще всего привести ее к жордановой форме. Как нетрудно проверить, для характеристических чисел $\lambda_{1}, \lambda_{2}, \lambda_{3}$ собственными являются соответственно векторы

$$
\begin{aligned}
& v_{1}=\left\{v_{11}, v_{12}, v_{13}\right\}=\{1,1,1\}, \\
& v_{2}=\left\{v_{21}, v_{22}, v_{23}\right\}=\left\{\Delta_{31}+q_{13} \lambda_{2}, \Delta_{32}+q_{23} \lambda_{2}, \lambda_{2}^{2}-\left(q_{11}+q_{22}\right) \lambda_{2}+\Delta_{33}\right\}, \\
& v_{3}=\left\{v_{31}, v_{32}, v_{33}\right\}=\left\{\Delta_{31}+q_{13} \lambda_{3}, \Delta_{32}+q_{23} \lambda_{3}, \lambda_{3}^{2}-\left(q_{11}+q_{22}\right) \lambda_{3}+\Delta_{33}\right\} .
\end{aligned}
$$

В рассматриваемом регулярном случае среди характеристических чисел нет равных, и преобразующая матрица

$$
V=\left(\begin{array}{lll}
1 & v_{21} & v_{31} \\
1 & v_{22} & v_{32} \\
1 & v_{23} & v_{33}
\end{array}\right)
$$

невырожденная. Ее определитель

$$
w=\operatorname{det} V=v_{31}\left(v_{23}-v_{22}\right)+v_{32}\left(v_{21}-v_{23}\right)+v_{33}\left(v_{22}-v_{21}\right)
$$

не равен нулю, и существует обратная матрица

$$
W=\frac{1}{w}\left(\begin{array}{lll}
w_{11} & w_{21} & w_{31} \\
w_{12} & w_{22} & w_{32} \\
w_{13} & w_{23} & w_{33}
\end{array}\right),
$$

где $w_{\beta \alpha}$ - алгебраическое дополнение элемента $v_{\beta \alpha}$ матрицы $V$, которое легко вычисляется ( $\alpha-$ номер строки, $\beta-$ номер столбца). Жорданова матрица

$$
J=\left(\begin{array}{ccc}
1 & 0 & 0 \\
0 & \lambda_{2} & 0 \\
0 & 0 & \lambda_{3}
\end{array}\right)
$$

диагональна, и для каждого $n \geqslant 0$ верно равенство

$$
Q^{n}=V J^{n} W
$$

Отсюда получаем общее выражение для элементов матрицы $Q^{n}$, равных вероятностям перехода из $\alpha$ в $\beta$ за $n$ шагов:

$$
q_{\alpha \beta}(n)=\mathbf{P}\{\xi(n)=\beta \mid \xi(0)=\alpha\}=\frac{w_{\beta 1}}{w}+\frac{w_{\beta 2}}{w} \lambda_{2}^{n} v_{2 \alpha}+\frac{w_{\beta 3}}{w} \lambda_{3}^{n} v_{3 \alpha} .
$$


Формула Перрона [4, §5.1], [12] дает

$$
q_{\alpha \beta}(n)=\frac{\Delta_{\beta \alpha}(1)}{\left(1-\lambda_{2}\right)\left(1-\lambda_{3}\right)}+\frac{\Delta_{\beta \alpha}\left(\lambda_{2}\right)}{\left(\lambda_{2}-1\right)\left(\lambda_{2}-\lambda_{3}\right)} \lambda_{2}^{n}+\frac{\Delta_{\beta \alpha}\left(\lambda_{3}\right)}{\left(\lambda_{3}-1\right)\left(\lambda_{3}-\lambda_{2}\right)} \lambda_{3}^{n},
$$

где $\Delta_{\beta \alpha}(\lambda)$ - алгебраическое дополнение $\beta \alpha$-элемента матрицы $Q(\lambda)=\lambda I-Q$. (Подчеркнем, что в формуле (2) индексы $\alpha$ и $\beta$ транспонированы.)

Как легко проверить,

$$
\begin{aligned}
& \Delta_{\beta \beta}(\lambda)=\lambda^{2}-\left(\tau-q_{\beta \beta}\right) \lambda+\Delta_{\beta \beta}, \\
& \Delta_{\beta \alpha}(\lambda)=\lambda q_{\alpha \beta}+\Delta_{\beta \alpha}, \quad \alpha \neq \beta .
\end{aligned}
$$

Общее выражение для матрицы $Q^{n}$ дает интерполяционная формула Лагранжа (см. раздел 5 в гл. 5 в [12]):

$$
Q^{n}=\frac{\left(Q-\lambda_{2} I\right)\left(Q-\lambda_{3} I\right)}{\left(1-\lambda_{2}\right)\left(1-\lambda_{3}\right)}+\frac{(Q-I)\left(Q-\lambda_{3} I\right)}{\left(\lambda_{2}-1\right)\left(\lambda_{2}-\lambda_{3}\right)} \lambda_{2}^{n}+\frac{(Q-I)\left(Q-\lambda_{2} I\right)}{\left(\lambda_{3}-1\right)\left(\lambda_{3}-\lambda_{2}\right)} \lambda_{3}^{n} .
$$

Из формулы Лагранжа следует эквивалентная ей формула Сильвестра:

$$
Q^{n}=\frac{1}{b\left(\lambda_{2}, \lambda_{3}\right)}\left(b_{0}\left(\lambda_{2}, \lambda_{3}, n\right) I-b_{1}\left(\lambda_{2}, \lambda_{3}, n\right) Q+b_{2}\left(\lambda_{2}, \lambda_{3}, n\right) Q^{2}\right),
$$

где

$$
\begin{aligned}
b\left(\lambda_{2}, \lambda_{3}\right) & =\left(1-\lambda_{2}\right)\left(1-\lambda_{3}\right)\left(\lambda_{2}-\lambda_{3}\right), \\
b_{0}\left(\lambda_{2}, \lambda_{3}, n\right) & =\left(1-\lambda_{3}\right)\left(\lambda_{2}-\lambda_{3}\right) \lambda_{2} \lambda_{3}-\left(1-\lambda_{3}\right) \lambda_{3} \lambda_{2}^{n}+\left(1-\lambda_{2}\right) \lambda_{2} \lambda_{3}^{n}, \\
b_{1}\left(\lambda_{2}, \lambda_{3}, n\right) & =\left(\lambda_{2}^{n}-\lambda_{3}^{n}\right)-\left(1-\lambda_{3}^{2}\right) \lambda_{2}^{n}+\left(1-\lambda_{2}^{2}\right) \lambda_{3}^{n}, \\
b_{2}\left(\lambda_{2}, \lambda_{3}, n\right) & =\left(\lambda_{2}-\lambda_{3}\right)-\left(1-\lambda_{3}\right) \lambda_{2}^{n}+\left(1-\lambda_{2}\right) \lambda_{3}^{n} .
\end{aligned}
$$

В формулах (2)-(4) не используются собственные векторы. Нужны только попарно различные характеристические числа матрицы $Q$.

2.3. В особом случае кратного корня

$$
\lambda=\lambda_{2}=\lambda_{3}=\sqrt{\Delta}<1
$$

формулу для $Q^{n}$ можно получить из (3) предельным переходом при $\lambda_{2} \rightarrow \lambda, \lambda$, $\lambda$ :

$$
\begin{aligned}
Q^{n}=\frac{1}{(1-\lambda)^{2}}\left((\lambda I-Q)^{2}+n \lambda^{n-1}(I-Q) Q-(n-1) \lambda^{n}\left(I-Q^{2}\right)\right. \\
\left.+(n-2) \lambda^{n+1}(I-Q)\right) .
\end{aligned}
$$

Из формулы (5) следует эквивалентная ей формула Сильвестра:

$$
Q^{n}=\frac{1}{(1-\lambda)^{2}}\left(b_{0}(\lambda, n) I-b_{1}(\lambda, n) Q+b_{2}(\lambda, n) Q^{2}\right),
$$

где

$$
\begin{aligned}
& b_{0}(\lambda, n)=\lambda^{2}-(n-1) \lambda^{n}+(n-2) \lambda^{n+1}, \\
& b_{1}(\lambda, n)=2 \lambda-n \lambda^{n-1}+(n-2) \lambda^{n+1}, \\
& b_{2}(\lambda, n)=1-n \lambda^{n-1}+(n-1) \lambda^{n} .
\end{aligned}
$$


Коэффициент $b_{\alpha}(\lambda, n)$ получается из отношения $b_{\alpha}\left(\lambda_{2}, \lambda_{3}, n\right) /\left(\lambda_{2}-\lambda_{3}\right)$ предельным переходом при $\lambda_{2} \rightarrow \lambda, \lambda_{3} \rightarrow \lambda$.

В рассматриваемом особом случае формула Перрона дает:

$$
q_{\alpha \beta}(n)=\frac{\Delta_{\beta \alpha}(1)}{(1-\lambda)^{2}}+\partial_{x}\left(\frac{x^{n} \Delta_{\beta \alpha}(x)}{x-1}\right)_{x \rightarrow \lambda} .
$$

Вычисляя производную, находим, что

$$
q_{\alpha \beta}(n)=\frac{\Delta_{\beta \alpha}(1)}{(1-\lambda)^{2}}-\frac{n \lambda^{n-1}}{1-\lambda}\left(\Delta_{\beta \alpha}(\lambda)+\frac{\lambda}{n}\left(\frac{\Delta_{\beta \alpha}(\lambda)}{1-\lambda}+\Delta_{\beta \alpha}(0, \lambda)+\Delta_{\beta \alpha}(\lambda, 0)\right)\right) .
$$

Здесь $\Delta_{\beta \alpha}(0, \lambda), \Delta_{\beta \alpha}(\lambda, 0)$ - определители, получающиеся из $\Delta_{\beta \alpha}(\lambda)$ соответственно заменой элементов 1-го и 2-го столбца на их производные, равные 1 или 0 . Использованное при вычислении производной равенство

$$
\partial_{x}\left(\Delta_{\beta \alpha}(x)\right)=\Delta_{\beta \alpha}(0, x)+\Delta_{\beta \alpha}(x, 0)
$$

следует из правила дифференцирования определителей.

Выбор подходящей формулы из (1)-(7) для анализа и вычислений зависит от рассматриваемого случая и применяемой методики.

2.4. Случайные величины $x_{\beta}(n), y_{\beta}(n)$ и $z_{\beta}(n)$, равные соответственно числу $\beta$-значений, числу $\beta$-серий и числу переходов $\beta \rightarrow \beta$ в последовательности $\xi$ на отрезке $[0, n]$, формально выражаются равенствами

$$
\begin{aligned}
& x_{\beta}(n)=\eta_{\beta}(0)+\sum_{k=1}^{n} \eta_{\beta}(k), \\
& y_{\beta}(n)=x_{\beta}(n)-z_{\beta}(n), \\
& z_{\beta}(n)=\sum_{k=1}^{n} \eta_{\beta}(k-1) \eta_{\beta}(k) .
\end{aligned}
$$

Здесь $\eta_{\beta}(k)$ обозначает индикатор события $\xi(k)=\beta$ :

$$
\eta_{\beta}(k)=1 \quad(\xi(k)=\beta), \quad \eta_{\beta}(k)=0 \quad(\xi(k) \neq \beta) .
$$

Для случайной величины $y_{\beta}(n)$ верно также равенство

$$
y_{\beta}(n)=\eta_{\beta}(0)+\sum_{k=1}^{n}\left(1-\eta_{\beta}(k-1)\right) \eta_{\beta}(k) .
$$

Сумма в (9) равна числу переходов $\alpha \rightarrow \beta(\alpha \neq \beta)$ в последовательности $\xi$ на отрезке $[0, n]$. Такой переход описывает начало $\beta$-серии. Переход $\beta \rightarrow \beta$ описывает продолжение $\beta$-серии. Последовательность $z_{\beta}(n)$ будет играть техническую роль. Последовательности $x_{\beta}(n)$ и $y_{\beta}(n)$ в общем случае немарковские.

\section{Задачи.}

(1) Найти распределения случайных переменных $\xi(n)$ и случайных величин $x_{\beta}(n)$, $y_{\beta}(n)$.

(2) Найти средние значения, дисперсии и ковариации случайных величин $x_{\beta}(n), y_{\beta}(n)$. 


\section{3. Распределение $\xi(k)$}

3.1. Пусть

$$
p_{\beta}(n)=\mathbf{P}\{\xi(n)=\beta\}, \quad P(n)=\left\{p_{1}(n), p_{2}(n), p_{3}(n)\right\} .
$$

По марковскому свойству $P(n)=A Q^{n}$. Из (1) получаем равенство

$$
p_{\beta}(n)=\frac{w_{\beta 1}}{w}+\left(\frac{w_{\beta 2}}{w} \sum_{\alpha=1}^{3} a_{\alpha} v_{2 \alpha}\right) \lambda_{2}^{n}+\left(\frac{w_{\beta 3}}{w} \sum_{\alpha=1}^{3} a_{\alpha} v_{3 \alpha}\right) \lambda_{3}^{n} .
$$

Равенство (2) дает

$$
p_{\beta}(n)=\frac{\Delta_{\beta}(1)}{\left(1-\lambda_{2}\right)\left(1-\lambda_{3}\right)}+\frac{\Delta_{\beta}\left(\lambda_{2}\right)}{\left(\lambda_{2}-1\right)\left(\lambda_{2}-\lambda_{3}\right)} \lambda_{2}^{n}+\frac{\Delta_{\beta}\left(\lambda_{3}\right)}{\left(\lambda_{3}-1\right)\left(\lambda_{3}-\lambda_{2}\right)} \lambda_{3}^{n},
$$

где

$$
\Delta_{\beta}(\lambda)=\sum_{\alpha=1}^{3} a_{\alpha} \Delta_{\beta \alpha}(\lambda)
$$

Как легко проверить,

$$
\Delta_{\beta}(\lambda)=a_{\beta} \lambda^{2}-\lambda\left(a_{\beta} \tau-A Q_{\beta}\right)+A M_{\beta},
$$

где $Q_{\beta}$ и $M_{\beta}$ обозначают $\beta$-столбцы матриц $Q$ и $M$.

3.2. В регулярном случае, когда $\left|\lambda_{2}\right|<1,\left|\lambda_{3}\right|<1$, существует предельное распределение и по (10), (11) предельные вероятности выражаются равенствами

$$
p_{\beta}=p_{\beta}(\infty)=\lim _{n \rightarrow \infty} p_{\beta}(n)=\frac{w_{\beta 1}}{w}=\frac{\Delta_{\beta}(1)}{\left(1-\lambda_{2}\right)\left(1-\lambda_{3}\right)} .
$$

В особом случае кратного корня $\lambda=\lambda_{2}=\lambda_{3}=\sqrt{\Delta}<1$ из (7) следует равенство

$$
p_{\beta}=\frac{\Delta_{\beta}(1)}{(1-\lambda)^{2}} \text {. }
$$

Другие особые случаи нужно рассматривать специально. Это нетрудно сделать.

Введем обозначения, которые будут использоваться для упрощения формул:

$$
\begin{aligned}
\rho & =\frac{1}{\lambda_{2}-\lambda_{3}}, \\
\varkappa & =\frac{1}{\left(\lambda_{2}-1\right)\left(\lambda_{3}-1\right)}=\frac{1}{(1-v)^{2}-\mu^{2}}, \\
c_{\beta}(\gamma, n) & =\frac{\Delta_{\beta}\left(\lambda_{2}\right) \lambda_{2}^{n}}{\left(\lambda_{2}-1\right)^{\gamma}}-\frac{\Delta_{\beta}\left(\lambda_{3}\right) \lambda_{3}^{n}}{\left(\lambda_{3}-1\right)^{\gamma}} \\
c_{\alpha \beta}(\gamma, n) & =\frac{\Delta_{\beta \alpha}\left(\lambda_{2}\right) \lambda_{2}^{n}}{\left(\lambda_{2}-1\right)^{\gamma}}-\frac{\Delta_{\beta \alpha}\left(\lambda_{3}\right) \lambda_{3}^{n}}{\left(\lambda_{3}-1\right)^{\gamma}} \\
d_{\beta}(\gamma, n) & =\frac{\Delta_{\beta}\left(\lambda_{2}\right) \Delta_{\beta \beta}\left(\lambda_{2}\right) \lambda_{2}^{n}}{\left(\lambda_{2}-1\right)^{\gamma}}-\frac{\Delta_{\beta}\left(\lambda_{3}\right) \Delta_{\beta \beta}\left(\lambda_{3}\right) \lambda_{3}^{n}}{\left(\lambda_{3}-1\right)^{\gamma}}, \\
d_{\beta \beta}(\gamma, n) & =\frac{\Delta_{\beta}\left(\lambda_{3}\right) \Delta_{\beta \beta}\left(\lambda_{2}\right) \lambda_{2}^{n}}{\left(\lambda_{2}-1\right)^{\gamma}}-\frac{\Delta_{\beta}\left(\lambda_{2}\right) \Delta_{\beta \beta}\left(\lambda_{3}\right) \lambda_{3}^{n}}{\left(\lambda_{3}-1\right)^{\gamma}},
\end{aligned}
$$




$$
d_{\beta \beta}(\gamma, m, n)=\frac{\Delta_{\beta}\left(\lambda_{2}\right) \Delta_{\beta \beta}\left(\lambda_{3}\right) \lambda_{2}^{m} \lambda_{3}^{n-m}}{\left(\lambda_{3}-1\right)^{\gamma}}-\frac{\Delta_{\beta}\left(\lambda_{3}\right) \Delta_{\beta \beta}\left(\lambda_{2}\right) \lambda_{3}^{m} \lambda_{2}^{n-m}}{\left(\lambda_{2}-1\right)^{\gamma}} .
$$

Из (2), (11) и (12) в рассматриваемом регулярном случае получаем $(n>0)$

$$
\begin{array}{ll}
p_{\beta}(0)=a_{\beta}, & q_{\beta \beta}(0)=1, \\
p_{\beta}(n)=p_{\beta}+\rho c_{\beta}(1, n), & q_{\alpha \beta}(n)=p_{\beta}+\rho c_{\alpha \beta}(1, n) .
\end{array}
$$

Подчеркнем, что в регулярном случае при любом $\alpha$ верно равенство $q_{\alpha \beta}(\infty)=p_{\beta}$ (см. гл. 1 в [4]).

3.3. Рассмотрим частный случай, когда переходы $\alpha \rightarrow \alpha$ имеют одну и ту же вероятность $p$, переходы $2,3 \rightarrow 1$ имеют одну и ту же вероятность $q=1-p$, переходы $1 \rightarrow 1,3$ имеют одну и ту же вероятность $r=q / 2$, переходы $2 \rightarrow 3,3 \rightarrow 2$ невозможны. В этом случае

$$
Q=\left(\begin{array}{lll}
p & r & r \\
q & p & 0 \\
q & 0 & p
\end{array}\right)
$$

Легко проверить равенства

$$
\begin{aligned}
\tau= & 3 p, \quad \Delta=p(p-q), \quad v=p-r, \quad \mu=r, \quad \lambda_{2}=p, \quad \lambda_{3}=p-q, \\
& p_{1}(n)=\frac{1}{2}\left(1+\left(a_{1}-\left(1-a_{1}\right)\right)(p-q)^{n}\right), \\
& p_{2}(n)=p_{3}(n)=\frac{1}{4}\left(1+2\left(a_{2}-a_{3}\right) p^{n}-\left(a_{1}-\left(1-a_{1}\right)\right)(p-q)^{n}\right) .
\end{aligned}
$$

Предельное распределение $\left(p_{1}, p_{2}, p_{3}\right)=(1 / 2,1 / 4,1 / 4)$ не зависит от начальных и переходных вероятностей.

\section{4. Средние значения}

4.1. Среднее значение $\mathbf{E}\left(x_{\beta}(n)\right)$ случайной величины $x_{\beta}(n)$ равно сумме вероятностей $p_{\beta}(n)=\mathbf{P}\left\{\eta_{\beta}(k)=1\right\}, n \geqslant 0$. Применяя равенство (13) и суммируя, в регулярном случае получаем, что

$$
\mathbf{E}\left(x_{\beta}(n)\right)=a_{\beta}+n p_{\beta}-\rho c_{\beta}(2,1)+\rho c_{\beta}(2, n+1) .
$$

Заметим, что в рассматриваемом регулярном случае

$$
\left(1-\lambda_{2}\right)\left(1-\lambda_{3}\right)=(1-v)^{2}-\mu^{2}=2+\Delta-\tau \neq 0 .
$$

Положим

$$
r_{\beta}(u, v)=(1-v)^{2}\left(a_{\beta} u^{2}-\left(a_{\beta} \tau-A Q_{\beta}\right) u+A M_{\beta}\right) .
$$

Здесь, как и прежде, $A=\left(a_{\beta}\right)$ - строка начальных вероятностей, а $Q_{\beta}$ и $M_{\beta}$ обозначают $\beta$-столбцы матриц $Q$ и $M$. Как нетрудно проверить,

$$
c_{\beta}(2, m)=\varkappa^{2}\left(r_{\beta}(\mu+v, v-\mu)(\mu+v)^{m}-r_{\beta}(v-\mu, \mu+v)(v-\mu)^{m}\right) .
$$

Для постоянного члена и остатка верны равенства

$$
\begin{aligned}
\rho c_{\beta}(2,1) & =\rho \varkappa^{2}\left(r_{\beta}(\mu+v, v-\mu)(\mu+v)-r_{\beta}(v-\mu, \mu+v)(v-\mu)\right), \\
\rho c_{\beta}(2, n+1) & =\rho \varkappa^{2}\left(r_{\beta}(\mu+v, v-\mu)(\mu+v)^{n+1}-r_{\beta}(v-\mu, \mu+v)(v-\mu)^{n+1}\right) .
\end{aligned}
$$


Замечание 2. В регулярном случае $\left|\lambda_{2}\right|=|v+\mu|<1,\left|\lambda_{3}\right|=|v-\mu|<1$, поэтому $\rho c_{\beta}(2, n+1)=O(\mu+|v|) \rightarrow 0$ при $n \rightarrow \infty$. Но в то же время $\rho \rightarrow \infty$ при $\lambda_{2}-\lambda_{3} \rightarrow 0$ и $x \rightarrow \infty$ при $\lambda_{2} \rightarrow 1$ или $\lambda_{3} \rightarrow 1$. Если характеристические числа близки к 1 или друг к другу, то лучше не переходить к общему знаменателю, дающему множители $\rho, x$, а использовать формулы с $b_{i}\left(\lambda_{2}, \lambda_{3}, n\right)$, которые нетрудно вывести.

4.2. Вычислим средние значения $\mathbf{E}\left(z_{\beta}(n)\right), \mathbf{E}\left(y_{\beta}(n)\right)$ случайных величин $z_{\beta}(n), y_{\beta}(n)$ при $n \geqslant 1$. При $k \geqslant 1$

$$
\mathbf{E}\left(\eta_{\beta}(k-1) \eta_{\beta}(k)\right)=\mathbf{P}\left\{\eta_{\beta}(k-1)=1, \eta_{\beta}(k)=1\right\}=p_{\beta}(k-1) q_{\beta \beta},
$$

поэтому

$$
\mathbf{E}\left(z_{\beta}(n)\right)=\sum_{k=1}^{n} \mathbf{E}\left(\eta_{\beta}(k-1) \eta_{\beta}(k)\right)=\sum_{k=1}^{n} p_{\beta}(k-1) q_{\beta \beta} .
$$

По аналогии с (14) находим, что

$$
\mathbf{E}\left(z_{\beta}(n)\right)=\left(n p_{\beta}-\rho c_{\beta}(2,0)+\rho c_{\beta}(2, n)\right) q_{\beta \beta} .
$$

Из (8), (14) и (15) следует равенство

$$
\begin{aligned}
\mathbf{E}\left(y_{\beta}(n)\right)=a_{\beta}+n p_{\beta}\left(1-q_{\beta \beta}\right)-\rho\left(c_{\beta}(2,1)-q_{\beta \beta} c_{\beta}(2,0)\right) & \\
& -\rho\left(c_{\beta}(2, n) q_{\beta \beta}-c_{\beta}(2, n+1)\right) .
\end{aligned}
$$

Для постоянных членов и остатков в (15) и (16) легко получить выражения, аналогичные полученным для (14). Относительно них верно замечание, аналогичное замечанию 2.

\section{5. Ковариации}

5.1. Вычислим ковариацию $\mathbf{C}\left(x_{\beta}(m), x_{\beta}(n)\right)$ случайных величин $x_{\beta}(m), x_{\beta}(n), m \leqslant n$. По определению,

$$
\mathbf{C}\left(x_{\beta}(m), x_{\beta}(n)\right)=\mathbf{E}\left(x_{\beta}(m) x_{\beta}(n)\right)-\mathbf{E}\left(x_{\beta}(m)\right) \mathbf{E}\left(x_{\beta}(n)\right) .
$$

С помощью (8) получаем равенства

$$
\mathbf{E}\left(x_{\beta}(m) x_{\beta}(n)\right)=\sum_{k=0}^{n} \sum_{j=0}^{m} \mathbf{E}\left(\eta_{\beta}(j) \eta_{\beta}(k)\right) .
$$

Заметим, что

$$
\mathbf{E}\left(x_{\beta}(m) x_{\beta}(n)\right)=a_{\beta}+S_{p}(m)+a_{\beta}\left(S_{q}(m)+S_{q}(n)\right)+S_{p q}(m, m)+S_{p q}(m, n),
$$

где

$$
\begin{gathered}
S_{p}(m)=\sum_{j=1}^{m} p_{\beta}(j), \quad S_{q}(m)=\sum_{j=1}^{m} q_{\beta \beta}(j), \\
S_{p q}(m, n)=\sum_{j=1}^{m} p_{\beta}(j) \sum_{i=1}^{n-j} q_{\beta \beta}(i)=\sum_{j=1}^{m} p_{\beta}(j) S_{q}(n-j) .
\end{gathered}
$$


Суммы $S_{p}(m)=S_{p_{\beta}}(m)$ и $S_{q}(m)=S_{q_{\beta}}(m)$ равны средним соответственно чисел значений $\beta$ и переходов $\beta \rightarrow \beta$ в последовательности $\xi$ на отрезке $[1, m]$. Используя (8), суммируя и приводя подобные члены, находим, что

$$
\begin{aligned}
S_{p}(m) & =m p_{\beta}-\rho c_{\beta}(2,1)+\rho c_{\beta}(2, m+1), \\
S_{q}(m) & =m p_{\beta}-\rho c_{\beta \beta}(2,1)+\rho c_{\beta \beta}(2, m+1), \\
S_{p q}(m, n) & =A S(m, n)+B S+R S(m, n),
\end{aligned}
$$

где

$$
\begin{aligned}
A S(m, n)= & n p_{\beta}\left(m p_{\beta}-\rho c_{\beta}(2,1)\right)-\frac{1}{2} m(m+1) p_{\beta}^{2}-m p_{\beta} \rho c_{\beta \beta}(2,1), \\
B S= & -\rho\left(p_{\beta} c_{\beta}(3,1)-\rho c_{\beta}(2,1) c_{\beta \beta}(2,1)\right), \\
R S(m, n)= & \rho R S 1(m, n)+\rho^{2} R S 2(m, n)+\rho^{3} R S 3(m, n), \\
R S 1(m, n)= & (n-m) p_{\beta} c_{\beta}(2, m+1)+p_{\beta}\left(c_{\beta}(3, m+1)\right. \\
& \left.\quad+c_{\beta \beta}(3, n+1)-c_{\beta \beta}(3, n-m+1)\right), \\
R S 2(m, n)= & m d_{\beta}(3, n+1)-c_{\beta}(2, m+1) c_{\beta \beta}(2,1), \\
R S 3(m, n)= & -\Delta \varkappa\left(d_{\beta \beta}(1, n)+d_{\beta \beta}(1, m, n)\right) .
\end{aligned}
$$

Подставляя равенства из групп (19), (21) в (18), находим, что

$$
\mathbf{E}\left(x_{\beta}(m) x_{\beta}(n)\right)=A_{\text {exx }}(m, n)+B_{\text {exx }}+R_{\text {exx }}(m, n),
$$

где

$$
\begin{aligned}
A_{\text {exx }}(m, n)= & (n-1) m p_{\beta}^{2}+n p_{\beta}\left(a_{\beta}-\rho c_{\beta}(2,1)\right) \\
& \quad+m p_{\beta}\left(1+a_{\beta}-\rho\left(c_{\beta}(2,1)+2 c_{\beta \beta}(2,1)\right)\right), \\
B_{\text {exx }}= & \left(a_{\beta}-\rho c_{\beta}(2,1)\right)\left(1-2 \rho c_{\beta \beta}(2,1)\right)-p_{\beta} \rho\left(2 c_{\beta}(3,1)+c_{\beta \beta}(3,1)\right), \\
R_{\text {exx }}(m, n)= & R 1_{\text {exx }}(m, n) \rho+R 2_{\text {exx }}(m, n) \rho^{2}+R 3_{\text {exx }}(m, n) \rho^{3}, \\
R 1_{\text {exx }}(m, n)= & (n-m) p_{\beta} c_{\beta}(2, m+1)+c_{\beta}(2, m+1) \\
& \quad+a_{\beta}\left(c_{\beta \beta}(2, m+1)+c_{\beta \beta}(2, n+1)\right)+p_{\beta}\left(2 c_{\beta}(3, m+1)\right. \\
& \left.\quad+c_{\beta \beta}(3, m+1)+c_{\beta \beta}(3, n+1)-c_{\beta \beta}(3, n-m+1)\right), \\
R 2_{\text {exx }}(m, n)= & m\left(d_{\beta}(3, m+1)+d_{\beta}(3, n+1)\right)-2 c_{\beta}(2, m+1) c_{\beta \beta}(2,1), \\
R 3_{\text {exx }}(m, n)= & \Delta x\left(d_{\beta \beta}(1, m)+d_{\beta \beta}(1, n)+d_{\beta \beta}(1, m, m)+d_{\beta \beta}(1, m, n)\right) .
\end{aligned}
$$

Для произведения средних, вследствие (14), получаем равенство

$$
\mathbf{E}\left(x_{\beta}(m)\right) \mathbf{E}\left(x_{\beta}(n)\right)=A_{\text {eex }}(m, n)+B_{\text {eex }}+R_{\text {eex }}(m, n),
$$

где

$$
\begin{aligned}
A_{\text {eex }}(m, n)= & n p_{\beta}\left(m p_{\beta}+a_{\beta}-\rho c_{\beta}(2,1)\right)+m p_{\beta}\left(a_{\beta}-\rho c_{\beta}(2,1)\right), \\
B_{\text {eex }}= & \left(a_{\beta}-\rho c_{\beta}(2,1)\right)^{2}, \\
R_{e e x}(m, n)= & R 1_{e e x}(m, n) \rho+R 2_{e e x}(m, n) \rho^{2} \\
R 1_{\text {eex }}(m, n)= & n p_{\beta} c_{\beta}(2, m+1)+m p_{\beta} c_{\beta}(2, n+1) \\
& \quad+a_{\beta}\left(c_{\beta}(2, m+1)+c_{\beta}(2, n+1)\right), \\
R 2_{e e x}(m, n)= & c_{\beta}(2, m+1) c_{\beta}(2, n+1) \\
& \quad-c_{\beta}(2,1)\left(c_{\beta}(2, m+1)+c_{\beta}(2, n+1)\right) .
\end{aligned}
$$


Вычитая (23) из (22), находим, что

$$
\mathbf{C}\left(x_{\beta}(m) x_{\beta}(n)\right)=A_{c x x}(m)+B_{c x x}+R_{c x x}(m, n),
$$

где

$$
\begin{aligned}
A_{c x x}(m)= & A_{e x x}(m, n)-A_{e e x}(m, n) \\
= & m p_{\beta}\left(1-p_{\beta}-2 \rho c_{\beta \beta}(2,1)\right), \\
B_{c x x}= & B_{e x x}-B_{e e x}=\left(a_{\beta}-\rho c_{\beta}(2,1)\right)\left(1-a_{\beta}+\rho\left(c_{\beta}(2,1)-2 c_{\beta \beta}(2,1)\right)\right) \\
& \quad-p_{\beta} \rho\left(2 c_{\beta}(3,1)+c_{\beta \beta}(3,1)\right), \\
R_{c x x}(m, n)= & R_{e x x}(m, n)-R_{\text {eex }}(m, n) .
\end{aligned}
$$

Как нетрудно проверить,

$$
2 \rho c_{\beta \beta}(2,1)=2 \varkappa^{2}\left(2(\Delta-v)\left(1-q_{\beta \beta}\right)+(\Delta-1)\left(\Delta-\Delta_{\beta \beta}\right)\right) .
$$

Подстановка этого выражения в (24) дает выражение коэффициента при главном члене ковариации $\mathbf{C}\left(x_{\beta}(m) x_{\beta}(n)\right)$ через след и определитель переходной матрицы $Q$, ее элементы и миноры. Точно так же можно получить развернутые выражения для постоянного члена $B_{c x x}$ и остатка $R_{c x x}(m, n)$. Заметим, что

$$
R_{c x x}(m, n)=\rho \varkappa O\left(m(\mu+\mid v)^{m} \mid\right) .
$$

Так как $\mathbf{C}\left(x_{\beta}(n) x_{\beta}(n)\right)=\mathbf{V}\left(x_{\beta}(n)\right)$, из (24) получается формула для дисперсии $\mathbf{V}\left(x_{\beta}(n)\right)$ случайной переменной $x_{\beta}(n)$ :

$$
\mathbf{V}\left(x_{\beta}(n)\right)=a_{\beta}\left(1-a_{\beta}\right)+n p_{\beta}\left(1-p_{\beta}-2 \rho c_{\beta \beta}(2,1)\right)-B_{v x}+R_{c x x}(n, n),
$$

где

$$
\begin{aligned}
B_{v x}=\rho\left(c_{\beta}(2,1)-2 a_{\beta}\left(c_{\beta}(2,1)-c_{\beta \beta}(2,1)\right)\right. & \left.+p_{\beta}\left(2 c_{\beta}(3,1)+c_{\beta \beta}(3,1)\right)\right) \\
& +\rho^{2}\left(c_{\beta}^{2}(2,1)-2 d_{\beta}(4,2)\right)+2 \rho^{3} d_{\beta \beta}(2,1) \Delta .
\end{aligned}
$$

Подставляя в это выражение известные значения $c_{\beta}(\gamma, m), c_{\beta \beta}(\gamma, m), d_{\beta}(\gamma, m), d_{\beta \beta}(\gamma, m)$, $\Delta_{\beta}(\lambda), \Delta_{\beta \beta}(\lambda)$ для $\lambda=\lambda_{2}=\mu+v, \lambda=\lambda_{3}=v-\mu$, легко выразить постоянный член $B_{v x}$ дисперсии $\mathbf{V}\left(x_{\beta}(n)\right)$ через след и определитель переходной матрицы $Q$, ее элементы и миноры. То же самое можно сделать и для остатка $R_{c x x}(n, n)$, но выражение получается слишком громоздким.

Если $m>n$, то

$$
\mathbf{C}\left(x_{\beta}(m) x_{\beta}(n)\right)=n p_{\beta}\left(1-p_{\beta}-2 \rho c_{\beta \beta}(2,1)\right)+B_{c x x}+R_{c x x}(n, m) .
$$

Это следует из симметричности проведенных при доказательстве равенства (24) рассуждений.

5.2. Вычислим ковариацию $\mathbf{C}\left(z_{\beta}(m), z_{\beta}(n)\right)$ случайных величин $z_{\beta}(m), z_{\beta}(n), m \leqslant n$. По определению,

$$
\mathbf{C}\left(z_{\beta}(m), z_{\beta}(n)\right)=\mathbf{E}\left(z_{\beta}(m) z_{\beta}(n)\right)-\mathbf{E}\left(z_{\beta}(m)\right) E\left(z_{\beta}(n)\right) .
$$


С помощью (8) получаем равенства

$$
\mathbf{E}\left(z_{\beta}(m) z_{\beta}(n)\right)=\sum_{k=1}^{n} \sum_{j=1}^{m} e_{\beta}(j, k),
$$

где

$$
e_{\beta}(j, k)=\mathbf{E}\left(\eta_{\beta}(j-1) \eta_{\beta}(j) \eta_{\beta}(k-1) \eta_{\beta}(k)\right) .
$$

Заметим, что

$$
\begin{aligned}
\mathbf{E}\left(z_{\beta}(m) z_{\beta}(n)\right)=( & \left.a_{\beta}+S_{p}(m-1)\right) q_{\beta \beta}\left(1+2 q_{\beta \beta}\right) \\
& +a_{\beta} q_{\beta \beta}^{2}\left(S_{q}(m-2)+S_{q}(n-2)\right) \\
& +q_{\beta \beta}^{2}\left(S_{p q}(m-1, m-2)\right. \\
& \left.+S_{p q}(m-1, n-2)\right) ;
\end{aligned}
$$

откуда, используя (19) и (21), получаем равенство

$$
\mathbf{E}\left(z_{\beta}(m) z_{\beta}(n)\right)=\left(A_{e z z}(m, n)+B_{e z z}-R_{e z z}(m, n)\right) q_{\beta \beta},
$$

где

$$
\begin{aligned}
A_{e z z}(m, n)= & p_{\beta}\left(m n p_{\beta} q_{\beta \beta}+n q_{\beta \beta}\left(a_{\beta}-p_{\beta}-\rho c_{\beta}(2,1)\right)\right) \\
& +m\left(1+q_{\beta \beta}\left(2+a_{\beta}-4 p_{\beta}-\rho\left(c_{\beta}(2,1)+2 c_{\beta \beta}(2,1)\right)\right)\right), \\
\left.B_{e z z}=a_{\beta}-p_{\beta}-\rho c_{\beta}(2,1)\right)\left(1+2 q_{\beta \beta}\left(1-2 p_{\beta}-\rho c_{\beta \beta}(2,1)\right)\right) & -p_{\beta} q_{\beta \beta} \rho\left(2 c_{\beta}(3,1)+c_{\beta \beta}(3,0)\right), \\
R_{e z z}(m, n)= & R 1_{e z z}(m, n) \rho+R 2_{e z z}(m, n) \rho^{2}+R 3_{e z z}(m, n) \rho^{3}, \\
R 1_{e z z}(m, n)=p_{\beta} q_{\beta \beta}(n-m) c_{\beta}(2, m)+\left(1+2 q \beta \beta-4 p_{\beta} q_{\beta \beta}\right) c_{\beta}(2, m) & \quad+a_{\beta} q_{\beta \beta}\left(c_{\beta \beta}(2, m-1)+c_{\beta \beta}(2, n-1)\right) \\
& +p_{\beta} q_{\beta \beta}\left(2 c_{\beta}(3, m+1)+c_{\beta \beta}(3, m-1)\right. \\
& \left.+c_{\beta \beta}(3, n-1)-c_{\beta \beta}(3, n-m)\right), \\
R 2_{e z z}(m, n)= & q_{\beta \beta}\left((m-1)\left(d_{\beta}(3, m-1)+d_{\beta}(3, n-1)\right)\right. \\
& \left.-2 c_{\beta}(2, m) c_{\beta \beta}(2,1)\right), \\
R 3_{e z z}(m, n)=\Delta & x q_{\beta \beta}\left(d_{\beta \beta}(1, m-2)+d_{\beta \beta}(1, n-2)\right. \\
& \left.+d_{\beta \beta}(1, m-1, m-2)+d_{\beta \beta}(1, m-1, n-2)\right) .
\end{aligned}
$$

Для произведения средних, вследствие (15), получаем выражение

$$
\mathbf{E}\left(z_{\beta}(m)\right) \mathbf{E}\left(z_{\beta}(n)\right)=\left(A_{e e z}(m, n)+B_{e e z}+R_{e e z}(m, n)\right) q_{\beta \beta}^{2},
$$

где

$$
\begin{aligned}
A_{e e z}(m, n) & =n m p_{\beta}^{2}-(m+n) p_{\beta} \rho c_{\beta}(2,0), \\
B_{e e z} & =\left(\rho c_{\beta}(2,0)\right)^{2}, \\
R_{e e z}(m, n) & =R 1_{e e z}(m, n) \rho+R 2_{e e z}(m, n) \rho^{2}, \\
R 1_{e e z}(m, n) & =m p_{\beta} c_{\beta}(2, n)+n p_{\beta} c_{\beta}(2, m), \\
R 2_{e e z}(m, n) & =c_{\beta}(2, m) c_{\beta}(2, n)-c_{\beta}(2,0)\left(c_{\beta}(2, m)+c_{\beta}(2, n)\right) .
\end{aligned}
$$


Вычитая (28) из (27), находим, что

$$
\mathbf{C}\left(z_{\beta}(m) z_{\beta}(n)\right)=\left(A_{c z z}(m)+B_{c z z}+R_{c z z}(m, n)\right) q_{\beta \beta},
$$

где

$$
\begin{aligned}
A_{c z z}(m)= & A_{e z z}(m, n)-A_{e e z}(m, n) q_{\beta \beta} \\
= & p_{\beta} m\left(1+q_{\beta \beta}\left(2-3 p_{\beta}-2 \rho c_{\beta \beta}(2,1)\right)\right), \\
B_{c z z}= & B_{e z z}-B_{e e z} q_{\beta \beta}=\left(a_{\beta}-p_{\beta}-\rho c_{\beta}(2,1)\right)\left(1+2 q_{\beta \beta}\left(1-2 p_{\beta}-\rho c_{\beta \beta}(2,1)\right)\right) \\
& \quad-q_{\beta \beta} \rho^{2} c_{\beta}^{2}(2,0)-p_{\beta} q_{\beta \beta} \rho\left(2 c_{\beta}(3,1)+c_{\beta \beta}(3,0)\right), \\
R_{c z z}(m, n)= & R_{e z z}(m, n)-R_{e e z}(m, n) q_{\beta \beta} .
\end{aligned}
$$

Так как $\mathbf{C}\left(z_{\beta}(n), z_{\beta}(n)\right)=\mathbf{V}\left(z_{\beta}(n)\right)$, из (29) получается формула для дисперсии $\mathbf{V}\left(z_{\beta}(n)\right)$ случайной переменной $z_{\beta}(n)$ :

$$
\mathbf{V}\left(z_{\beta}(n)\right)=\left(n p_{\beta}\left(1+\left(2-3 p_{\beta}-2 \rho c_{\beta \beta}(2,1)\right) q_{\beta \beta}\right)+B_{c z z}+R_{c z z}(n, n)\right) q_{\beta \beta} .
$$

Подставляя последовательно в выражение для $B_{c z z}$ известные значения входящих в него переменных, можно выразить постоянный член дисперсии $\mathbf{V}\left(z_{\beta}(n)\right)$ через след и определитель переходной матрицы $Q$, ее элементы и миноры. Выражение получается громоздким, но вычислительная процедура достаточно проста. То же самое можно сказать и об остатке $R_{c z z}(n, n)$ при не слишком больших значениях $n$.

5.3. Вычислим ковариацию $\mathbf{C}\left(x_{\beta}(m), z_{\beta}(n)\right)$ случайных величин $x_{\beta}(m), z_{\beta}(n), m \leqslant n$. Из определений следует, что

$$
\mathbf{C}\left(x_{\beta}(m), z_{\beta}(n)\right)=\mathbf{E}\left(x_{\beta}(m), z_{\beta}(n)\right)-\mathbf{E}\left(x_{\beta}(m)\right) \mathbf{E}\left(z_{\beta}(n)\right) .
$$

С помощью (8) получаем

$$
\mathbf{E}\left(x_{\beta}(m), z_{\beta}(n)\right)=\sum_{k=0}^{m} \sum_{j=1}^{n} \mathbf{E}\left(\eta_{\beta}(k) \eta_{\beta}(j-1) \eta_{\beta}(j)\right) .
$$

Как нетрудно проверить,

$$
\begin{aligned}
\mathbf{E}\left(x_{\beta}(m), z_{\beta}(n)\right)=\left(2 \left(a_{\beta}\right.\right. & \left.+S_{p}(m-1)\right)+a_{\beta}\left(S_{q}(m-1)+S_{q}(n-1)\right) \\
& \left.+S_{p q}(m, n-1)-S_{p q}(m, m-1)+2 S_{p q}(m-2, m-1)\right) q_{\beta \beta},
\end{aligned}
$$

откуда, используя (19) и (21), получаем равенство

$$
\mathbf{E}\left(x_{\beta}(m) z_{\beta}(n)\right)=\left(A_{\text {exz }}(m, n)+B_{\text {exz }}-R_{e x z}(m, n)\right) q_{\beta \beta},
$$

где

$$
\begin{aligned}
A_{e x z}(m, n)= & p_{\beta}\left(m n p_{\beta}+n\left(a_{\beta}-\rho c_{\beta}(2,1)\right)\right. \\
& \left.+m\left(2\left(1-p_{\beta}\right)-\rho\left(c_{\beta}(2,0)+2 c_{\beta \beta}(2,1)\right)\right)\right), \\
B_{e x z}=- & \rho\left(\left(2 c_{\beta}(3,0)+c_{\beta \beta}(3,1)+c_{\beta \beta}(2,0)\right) p_{\beta}+2 c_{\beta}(2,0)\right) \\
& +2 \rho^{2} c_{\beta}(2,0) c_{\beta \beta}(2,1),
\end{aligned}
$$




$$
\begin{aligned}
R_{\text {exz }}(m, n)= & R 1_{\text {exz }}(m, n) \rho+R 2_{\text {exz }}(m, n) \rho^{2}+R 3_{\text {exz }}(m, n) \rho^{3}, \\
R 1_{\text {exz }}(m, n)= & p_{\beta}(n-m) c_{\beta}(2, m+1)+a_{\beta}\left(c_{\beta \beta}(2, n)+c_{\beta \beta}(2, m)\right) \\
& \quad+2 c_{\beta}(2, m)-p_{\beta}\left(2 c_{\beta}(2, m-1)-c_{\beta \beta}(3, n)-3 c_{\beta \beta}(3, m)\right. \\
& \left.\quad+c_{\beta \beta}(3, n-m)\right), \\
R 2_{e x z}(m, n)= & m\left(d_{\beta}(3, n)+d_{\beta}(3, m)\right)-4 d_{\beta}(3, m)-2 c_{\beta}(2, m-1) c_{\beta \beta}(2,1), \\
R 3_{e x z}(m, n)=- & \Delta x\left(d_{\beta \beta}(1, n-1)+d_{\beta \beta}(1, m-1)+d_{\beta \beta}(1, m, n-1)\right. \\
& \left.-d_{\beta \beta}(1, m, m-1)+2 d_{\beta \beta}(1, m-2, m-1)\right) .
\end{aligned}
$$

Для произведения средних, вследствие (14), (15), получаем равенство

$$
\mathbf{E}\left(x_{\beta}(m)\right) \mathbf{E}\left(z_{\beta}(n)\right)=\left(A_{\text {eexz }}(m, n)+B_{\text {eexz }}+R_{\text {eexz }}(m, n)\right) q_{\beta \beta},
$$

где

$$
\begin{aligned}
A_{\text {eexz }}(m, n) & =n(m+1) p_{\beta}^{2}-(n+m) p_{\beta} \rho c_{\beta}(2,0), \\
B_{\text {eexz }} & =-\rho c_{\beta}(2,0)\left(a_{\beta}-\rho c_{\beta}(2,1)\right), \\
R_{\text {eexz }}(m, n) & =R 1_{\text {eexz }}(m, n) \rho+R 2_{\text {eexz }}(m, n) \rho^{2}, \\
R 1_{\text {eexz }}(m, n) & =m p_{\beta} c_{\beta}(2, n)+n p_{\beta} c_{\beta}(2, m+1)+a_{\beta} c_{\beta}(2, n), \\
R 2_{\text {eexz }}(m, n) & \left.=c_{\beta}(2, m+1) c_{\beta}(2, n)-c_{\beta}(2,0) c_{\beta}(2, m+1)-c_{\beta}(2,1) c_{\beta}(2, n)\right) .
\end{aligned}
$$

Вычитая (32) из (31), находим, что

$$
\mathbf{C}\left(x_{\beta}(m) z_{\beta}(n)\right)=\left(A_{c x z}(m)+B_{c x z}+R_{c x z}(m, n)\right) q_{\beta \beta}, \quad m \leqslant n,
$$

где

$$
\begin{aligned}
A_{c x z}(m)= & A_{e x z}(m, n)-A_{e x z}(m, n)=2 p_{\beta} m\left(1-p_{\beta}-\rho c_{\beta \beta}(2,1)\right), \\
B_{c x z}= & B_{e x z}-B_{e e x z}=-\rho\left(p_{\beta}\left(2 c_{\beta}(3,0)-c_{\beta}(2,0)+c_{\beta \beta}(3,0)+2 c_{\beta \beta}(2,0)\right)\right. \\
& \left.\quad+c_{\beta}(2,0)\left(2+\rho\left(c_{\beta}(2,0)-2 c_{\beta \beta}(2,1)\right)\right)\right), \\
R_{c x z}(m, n)= & R_{e x z}(m, n)-R_{e e x z}(m, n) .
\end{aligned}
$$

В случае $n<m$ справедливо равенство

$$
\begin{aligned}
\mathbf{E}\left(x_{\beta}(m) z_{\beta}(n)\right)= & \left(2\left(a_{\beta}+S_{p}(n-1)\right)+a_{\beta}\left(S_{q}(m-1)+S_{q}(n-1)\right)\right. \\
& \left.+S_{p q}(n-1, m-1)-S_{p q}(n-1, n-1)+2 S_{p q}(n-2, n-1)\right) q_{\beta \beta} ;
\end{aligned}
$$

отсюда, используя (19) и (21), получаем, что

$$
\mathbf{E}\left(x_{\beta}(m) z_{\beta}(n)\right)=\left(A_{e z x}(m, n)+B_{e z x}-R_{e z x}(m, n)\right) q_{\beta \beta},
$$

где

$$
\begin{aligned}
A_{e z x}(m, n)= & p_{\beta}\left(m n p_{\beta}+n\left(2-p_{\beta}-\rho\left(c_{\beta}(2,0)+2 c_{\beta \beta}(2,1)\right)\right)-m \rho c_{\beta}(2,0)\right), \\
B_{e z x}= & -\rho\left(2 c_{\beta}(2,0)+2 p_{\beta} c_{\beta}(3,0)+p_{\beta} c_{\beta \beta}(3,1)-2 \rho c_{\beta}(2,0) c_{\beta \beta}(2,1)\right), \\
R_{e z x}(m, n)= & R 1_{e z x}(m, n) \rho+R 2_{e z x}(m, n) \rho^{2}+R 3_{e z x}(m, n) \rho^{3}, \\
R 1_{e z x}(m, n)= & p_{\beta}(m-n) c_{\beta}(2, n)+a_{\beta}\left(c_{\beta \beta}(2, n)+c_{\beta \beta}(2, m)\right)+2 c_{\beta}(2, n) \\
& \quad+p_{\beta}\left(2 c_{\beta}(3, n)+c_{\beta \beta}(3, n)+c_{\beta \beta}(3, m)-c_{\beta \beta}(3, m-n+1)\right), \\
R 2_{e z x}(m, n)= & (n-1)\left(d_{\beta}(3, n)+d_{\beta}(3, m)\right)-2 d_{\beta}(3, n)-2 c_{\beta}(2, n-1) c_{\beta \beta}(2,1), \\
R 3_{e z x}(m, n)= & -\Delta x\left(d_{\beta \beta}(1, m-1)+d_{\beta \beta}(1, n-1)+d_{\beta \beta}(1, n-1, m-1)\right. \\
& \left.\quad-d_{\beta \beta}(1, n-1, n-1)+2 d_{\beta \beta}(1, n-2, n-1)\right) .
\end{aligned}
$$


Вычитая (32) из (34), находим, что

$$
\mathbf{C}\left(x_{\beta}(m) z_{\beta}(n)\right)=\left(A_{c z x}(n)+B_{c z x}+R_{c z x}(m, n)\right) q_{\beta \beta}, \quad m>n,
$$

где

$$
\begin{aligned}
A_{c z x}(n)= & A_{e z x}(m, n)-A_{e e x z}(m, n)=2 p_{\beta} n\left(1-p_{\beta}-\rho c_{\beta \beta}(2,1)\right), \\
B_{c z x}= & B_{e z x}-B_{\text {eexz }}=-\rho\left(p_{\beta}\left(2 c_{\beta}(3,0)-c_{\beta}(2,0)+c_{\beta \beta}(3,1)\right)\right. \\
& \left.\quad+c_{\beta}(2,0)\left(2+\rho\left(c_{\beta}(2,0)-2 c_{\beta \beta}(2,1)\right)\right)\right), \\
R_{c z x}(m, n)= & R_{e z x}(m, n)-R_{e e x z}(m, n) .
\end{aligned}
$$

Заметим, что $A_{c z x}(n)=A_{c x z}(n), B_{c z x}=B_{c x z}+\rho p_{\beta} c_{\beta \beta}(2,0)$. Слагаемое $\rho p_{\beta} c_{\beta \beta}(2,0)=$ $\rho p_{\beta}\left(c_{\beta \beta}(3,1)-c_{\beta \beta}(3,0)\right)$ выделяется из членов $R 1_{e x z}(m, n), R 1_{e z x}(m, n)$ при $m=n$.

5.4. Вычислим ковариацию $\mathbf{C}\left(x_{\beta}(m), y_{\beta}(n)\right)$ случайных величин $x_{\beta}(m), y_{\beta}(n), m \leqslant n$. Так как $y_{\beta}(n)=x_{\beta}(n)-z_{\beta}(n)$, справедливо равенство

$$
\mathbf{C}\left(x_{\beta}(m), y_{\beta}(n)\right)=\mathbf{C}\left(x_{\beta}(m), x_{\beta}(n)\right)-\mathbf{C}\left(x_{\beta}(m), z_{\beta}(n)\right) .
$$

Подставляя значения ковариаций справа из равенств (24), (33) и приводя подобные члены, получаем, что

$$
\mathbf{C}\left(x_{\beta}(m), y_{\beta}(n)\right)=m p_{\beta}\left(\left(1-p_{\beta}\right)\left(1-2 q_{\beta \beta}\right)-2 \rho c_{\beta \beta}(2,1)\left(1-q_{\beta \beta}\right)\right)+B_{c x y}+R_{c x y}(m, n),
$$

где $B_{c x y}=B_{c x x}-B_{c x z} q_{\beta \beta}, R_{c x y}(m, n)=R_{c x x}(m, n)-R_{c x z}(m, n) q_{\beta \beta}$.

Пусть теперь $m>n$. Тогда, как нетрудно проверить,

$$
\mathbf{C}\left(x_{\beta}(m), y_{\beta}(n)\right)=n p_{\beta}\left(\left(1-p_{\beta}\right)\left(1-2 q_{\beta \beta}\right)-2 \rho c_{\beta \beta}(2,1)\left(1-q_{\beta \beta}\right)\right)+B_{c y x}+R_{c y x}(m, n),
$$

где $B_{c y x}=B_{c x x}-B_{c z x} q_{\beta \beta}, R_{c y x}(m, n)=R_{c x x}(m, n)-R_{c z x}(m, n) q_{\beta \beta}$. Несимметричность равенств (36) и (37) в слагаемых, не включающих главный член, объясняется различной структурой рассматриваемых переменных, описываемой равенствами (8) и формулами раздела 5.3 .

5.5. Вычислим ковариацию $\mathbf{C}\left(y_{\beta}(m), y_{\beta}(n)\right)$ случайных величин $y_{\beta}(m), y_{\beta}(n), m \leqslant n$. Из определений следует, что

$$
\begin{aligned}
\mathbf{C}\left(y_{\beta}(m), y_{\beta}(n)\right) \\
\quad=\mathbf{C}\left(x_{\beta}(m), x_{\beta}(n)\right)+\mathbf{C}\left(z_{\beta}(m), z_{\beta}(n)\right)-\mathbf{C}\left(x_{\beta}(m), z_{\beta}(n)\right)-\mathbf{C}\left(z_{\beta}(m), x_{\beta}(n)\right) .
\end{aligned}
$$

Подставляя известные значения ковариаций из равенств (24), (29), (33), (35) и приводя подобные члены, находим, что

$$
\begin{aligned}
\mathbf{C}\left(y_{\beta}(m), y_{\beta}(n)\right)= & m p_{\beta}\left(1-q_{\beta \beta}\right)\left(1-2 q_{\beta \beta}\right. \\
& \left.-p_{\beta}\left(1-3 q_{\beta \beta}\right)-2\left(1-q_{\beta \beta}\right) \rho c_{\beta \beta}(2,1)\right)+B_{c y y}+R_{c y y}(m, n),
\end{aligned}
$$

где

$$
\begin{aligned}
B_{c y y} & =B_{c x x}+\left(B_{c z z}-B_{c x z}-B_{c z x}\right) q_{\beta \beta} \\
R_{c x y}(m, n) & =R_{c x x}(m, n)+\left(R_{c z z}(m, n)-R_{c x z}(n, m)-R_{c z x}(m, n)\right) q_{\beta \beta} .
\end{aligned}
$$


Так как $\mathbf{C}\left(y_{\beta}(n), y_{\beta}(n)\right)=\mathbf{V}\left(y_{\beta}(n)\right)$, из (38) получается формула для дисперсии $\mathbf{V}\left(y_{\beta}(n)\right)$ случайной переменной $y_{\beta}(n)$ :

$$
\begin{aligned}
& \mathbf{V}\left(y_{\beta}(n)\right)=n p_{\beta}\left(1-q_{\beta \beta}\right)\left(1-2 q_{\beta \beta}-p_{\beta}\left(1-3 q_{\beta \beta}\right)-2\left(1-q_{\beta \beta}\right) \rho c_{\beta \beta}(2,1)\right) \\
&+B_{c y y}+R_{c y y}(n, n) .
\end{aligned}
$$

Подставляя последовательно в (39) в выражение для $B_{c y y}$ известные значения входящих в него переменных можно выразить постоянный член дисперсии $\mathbf{V}\left(y_{\beta}(n)\right)$ через след и определитель переходной матрицы $Q$, ее элементы и миноры.

\section{6. Близкие двоичным последовательности}

Введенные условия регулярности не позволяют прямо получить двоичную последовательность как частный случай троичной. Поэтому рассматриваются троичные последовательности, близкие двоичным. Они позволяют осуществить переход и представляют определенный самостоятельный интерес.

6.1. Рассмотрим близкую двоичной троичную марковскую последовательность с переходной матрицей

$$
Q=\left(\begin{array}{ccc}
q_{11} & 1-q_{11} & 0 \\
1-q_{22} & q_{22} & 0 \\
0 & 1-q_{33} & q_{33}
\end{array}\right)=\left(\begin{array}{ccc}
p & 1-p & 0 \\
1-q & q & 0 \\
0 & 1-r & r
\end{array}\right)
$$

При $a_{3}=0, q_{33}=1$ последовательность превращается в двоичную марковскую последовательность, исследовавшуюся в [1] с помощью производящих функций. Соответствие в обозначениях устанавливается с помощью соотношений раздела 2.1 и равенств $q_{11}=p$, $q_{22}=q, q_{33}=r$. Двоичную последовательность можно считать предельным случаем близкой троичной при $r \rightarrow 1$.

Чтобы исключить особые случаи, будем предполагать, что $0<q_{11}, q_{22}<1$ и $q_{11}+q_{22}<q_{33}<1$. При этом предположении характеристические числа переходной матрицы выражаются равенствами $\lambda_{1}=1, \lambda_{2}=q_{33}, \lambda_{3}=q_{11}+q_{22}-1$. Рассматриваемая троичная марковская последовательность регулярна, и для нее верны все полученные для регулярных последовательностей формулы. Формулы для близкой к двоичной последовательности получаются из них подстановкой значений $q_{13}=0, q_{23}=0, q_{31}=0$, $q_{12}=1-q_{11}, q_{21}=1-q_{22}, q_{32}=1-q_{33}$. Предельные вероятности для значений $\beta=1,2,3$ соответственно равны

$$
p_{1}=\frac{1-q_{22}}{1-\lambda_{3}}, \quad p_{2}=\frac{1-q_{11}}{1-\lambda_{3}}, \quad p_{3}=0 .
$$

Матрица переходных вероятностей за $n \geqslant 0$ шагов имеет вид

$$
Q=\left(\begin{array}{ccc}
p_{1}+p_{2} \lambda_{3}^{n} & p_{2}\left(1-\lambda_{3}^{n}\right) & 0 \\
p_{1}\left(1-\lambda_{3}^{n}\right) & p_{2}+p_{1} \lambda_{3}^{n} & 0 \\
p_{1}+a \lambda_{3}^{n}-b q_{33}^{n} & 1-p_{1}-a \lambda_{3}^{n}-(1-b) q_{33}^{n} & q_{33}^{n}
\end{array}\right)
$$

где $a=\rho p_{1}\left(1-q_{33}\right), b=\rho\left(1-q_{22}\right)$. При $n=0$ получается единичная матрица.

Вероятности значений $\beta=1,2,3$ в момент $n \geqslant 0$ соответственно равны

$$
p_{1}(n)=p_{1}+c \lambda_{3}^{n}-a_{3} b q_{33}^{n}, \quad p_{2}(n)=p_{2}-c \lambda_{3}^{n}-a_{3}(1-b) q_{33}^{n}, \quad p_{3}(n)=a_{3} q_{33}^{n},
$$


где $c=a_{1} p_{2}-a_{2} p_{1}+a_{3} \rho p_{1}\left(1-q_{33}\right)$. В частности $p_{1}(0)=a_{1}, p_{2}(0)=a_{2}, p_{3}(0)=a_{3}$. Для двоичной последовательности при $a_{3}=0$, используя равенства

$$
p_{1}+p_{2}=1, \quad a_{1} p_{2}-a_{2} p_{1}=a_{1}-p_{1}+a_{3} p_{1}=-\left(a_{2}-p_{2}\right)-a_{3} p_{2},
$$

после некоторых преобразований получаем такие же, как в [1], равенства

$$
p_{1}(n)=p_{1}+\left(a_{1}-p_{1}\right) \lambda_{3}^{n}, \quad p_{2}(n)=p_{2}+\left(a_{2}-p_{2}\right) \lambda_{3}^{n} .
$$

6.2. Средние значения рассматриваемых переменных для близкой двоичной последовательности выражаются равенствами, конкретизирующими общие равенства в разделах 4.1-4.2. Их легко получить и прямым суммированием вычисленных вероятностей, преобразуя так, чтобы выделялись члены с $a_{3}$. Суммирование вероятностей $p_{1}(n), p_{2}(n)$, $p_{3}(n)$ дает для средних чисел $\mathbf{E}\left(x_{\beta}(n)\right)$ значений $\beta=1,2,3$ равенства

$$
\begin{aligned}
& \mathbf{E} x_{1}(n)=(n+1) p_{1}+c \frac{1-\lambda_{3}^{n+1}}{1-\lambda_{3}}-a_{3} b \frac{1-\lambda_{2}^{n+1}}{1-\lambda_{2}}, \\
& \mathbf{E} x_{2}(n)=(n+1) p_{2}-c \frac{1-\lambda_{3}^{n+1}}{1-\lambda_{3}}-a_{3}(1-b) \frac{1-\lambda_{2}^{n+1}}{1-\lambda_{2}}, \\
& \mathbf{E} x_{3}(n)=a_{3} \frac{1-\lambda_{2}^{n+1}}{1-\lambda_{2}} .
\end{aligned}
$$

При $\beta=1, a_{3}=0$ и соответствующем изменении обозначений получается выведенное в [1] равенство для среднего числа единиц в двоичной последовательности. Средние числа $\mathbf{E}\left(z_{\beta}(n)\right), \mathbf{E}\left(y_{\beta}(n)\right)$ сохранений и серий значений $\beta=1,2,3$ при $n>0$ получаются из $\mathbf{E}\left(x_{\beta}(n)\right)$ по формулам

$$
\mathbf{E}_{z_{\beta}}(n)=\mathbf{E} x_{\beta}(n-1) q_{\beta \beta}, \quad \mathbf{E} y_{\beta}(n)=\mathbf{E} x_{\beta}(n-1)\left(1-q_{\beta \beta}\right)+p_{\beta}(n) .
$$

Из определений следует, что $\mathbf{E} z_{\beta}(0)=0, \mathbf{E} y_{\beta}(0)=a_{\beta}$.

6.3. Ковариации рассматриваемых переменных для близкой двоичной последовательности выражаются равенствами, конкретизирующими общие равенства в разделах 5.1-5.5. В качестве примера выпишем формулу для $\mathbf{C}\left(x_{1}(m), x_{1}(n)\right)$. Введем дополнительные обозначения

$$
\begin{gathered}
c_{1}(\lambda)=-\frac{1-q_{11}}{\rho(1-\lambda)^{2}}, \quad c_{2}(\lambda)=\frac{1-q_{22}}{\rho(1-\lambda)^{2}}, \quad c_{3}(\lambda)=\frac{p_{1}}{\varkappa(1-\lambda)^{2}}, \\
\Lambda(\gamma, m)=\rho\left(1-\lambda_{2}\right)\left(c_{2}\left(\lambda_{2}\right)\left(1-\lambda_{2}\right)^{\gamma} \lambda_{2}^{m}-c_{2}\left(\lambda_{3}\right)\left(1-\lambda_{3}\right)^{\gamma} \lambda_{3}^{m}\right) .
\end{gathered}
$$

Подстановка переходных вероятностей $q_{13}=0, q_{23}=0, q_{31}=0, q_{12}=1-q_{11}$, $q_{21}=1-q_{22}, q_{32}=1-q_{33}$ в общие формулы дает для $\beta=1$ равенства

$$
\begin{aligned}
r_{1}\left(\lambda_{2}, \lambda_{3}\right) & =a_{3} c_{3}\left(\lambda_{3}\right), \\
r_{1}\left(\lambda_{3}, \lambda_{2}\right) & =\frac{1}{\varkappa^{2}}\left(a_{1} c_{1}\left(\lambda_{3}\right)+a_{2} c_{2}\left(\lambda_{3}\right)\right)+a_{3} c_{3}\left(\lambda_{2}\right), \\
c_{1}(\gamma, m) & =(-1)^{\gamma}\left(c_{12}\left(\lambda_{3}\right)\left(1-\lambda_{3}\right)^{2-\gamma} \lambda_{3}^{m}+a_{3} \Lambda(2-\gamma, m)\right), \\
c_{11}(\gamma, m) & =(-1)^{\gamma} c_{1}\left(\lambda_{3}\right)\left(1-\lambda_{3}\right)^{2-\gamma} \lambda_{3}^{m}, \\
d_{1}(4,2) & =c_{1}\left(\lambda_{3}\right)\left(c_{12}\left(\lambda_{3}\right)-a_{3} c_{3}\left(\lambda_{3}\right) \frac{1}{\left(1-\lambda_{3}\right)^{4}}\right) \lambda_{3}^{2}, \\
d_{11}(2,1) & =a_{3} \varkappa^{2} \rho^{-2}\left(1-q_{11}\right)\left(1-q_{22}\right)\left(1-q_{33}\right) .
\end{aligned}
$$


Используя (24), получаем равенства

$$
\begin{gathered}
\mathbf{C}\left(x_{1}(m), x_{1}(n)\right)=A(m)+B+R(m, n), \\
A(m)=a_{1}\left(1-a_{1}\right)+m p_{1}\left(1-p_{1}\right) r_{33}, \quad r_{33}=\frac{1+\lambda_{3}}{1-\lambda_{3}}, \quad B=-\rho\left(B_{c}-B_{d}\right),
\end{gathered}
$$

где

$$
\begin{aligned}
& B_{c}=c_{1}(2,1)+\rho c_{1}(2,1)^{2}-2 a_{1}\left(c_{1}(2,1)-c_{11}(2,1)\right)+p_{1}\left(2 c_{1}(3,1)+c_{11}(3,1)\right), \\
& B_{d}=2 \rho\left(d_{1}(4,2)-\rho \Delta d_{11}(2,1)\right)
\end{aligned}
$$

с явно выписанными значениями $c_{1}(\gamma, m), c_{11}(\gamma, m), d_{1}(4,2), d_{11}(2,1)$. Остаточный член $R(m, n)$ получается подстановкой упрощенных переходных вероятностей в $R_{c x x}(m, n)$. Формула для двоичной последовательности получается при $a_{3}=0$ и $a_{2}=1-a_{1}$.

Точно так же подстановка переходных вероятностей $q_{13}=0, q_{23}=0, q_{31}=0$, $q_{12}=1-q_{11}, q_{21}=1-q_{22}, q_{32}=1-q_{33}$ в общие формулы позволяет получить для близкой двоичной последовательности формулы ковариаций всех других рассматриваемых переменных.

\section{Список литературы}

1. Савельев Л. Я., Балакин С. В., Совместное распределение числа единиц и числа 1-серий в двоичной марковской последовательности. Дискретная математика (2004) 16, №3, 43-62.

2. Марков А. А., Исчисление вероятностей. Типография Императорской Академии наук, СанктПетербург, 1913.

3. Колмогоров А. Н., Теория вероятностей и математическая статистика. Наука, Москва, 1986.

4. Романовский В. И., Дискретные иепи Маркова. Гостехиздат, Москва, 1949.

5. Феллер В., Введение в теорию вероятностей и ее приложения, 1. Мир, Москва, 1984.

6. Савельев Л. Я., Случайные соответствия, двоичные матрицы и серии. Дискретная математика (1999) 11, №4, 3-26.

7. Uchida M., On number of occurrences of success runs of specified length in a higher-order two-state Markov chain. Ann. Inst. Statist. Math. (1998) 50, 587-601.

8. Смирнов Н. В., Сарманов О. В., Захаров В. К., Локальная предельная теорема для чисел переходов в цепи Маркова и ее применения. Доклады АН СССР (1966) 167, №6, 1238-1241.

9. Сарманов О. В., Захаров В. К., Комбинаторная задача Н. В. Смирнова. Доклады АН СССР (1967) 176, №3, 530-532.

10. Захаров В. К., Сарманов О. В., О законе распределения числа серий в однородной цепи Маркова. Доклады АН СССР (1968) 179, №3, 526-528.

11. Гершгорин С. А., Über die Abgrenzung der Eigenwerte einer Matrix. Известия АН СССР, ОМЕН, сер. VII (1931) 749-754.

12. Гантмахер Ф. Р., Теория матриц. Наука, Москва, 1967.

Статья поступила 16.11.2010. 